\title{
Analysis of Influence of Directional Coupler on Power Calibration Results
}

\author{
Shenxiang Gao, Guiwei Long, Cui Bao \\ China Satellite Maritime Tracking and Control Department, Jiangyin, 214431, China
}

Key words: medium power calibration directional coupler measurement uncertainty

\begin{abstract}
The influence of the directional coupler on the power calibration results is analyzed synthetically. The calculation method of the coupling parameters and the calculation formula of the equivalent reflection coefficient are given. The coupling parameters of the coupler and the uncertainty of the introduction of the reflection parameters are quantitatively calculated. The reasons for the dispersion of the measured data are analyzed qualitatively.
\end{abstract}

\section{Introduction}

Generally less than $10 \mathrm{~mW}$ of radio frequency / microwave power called low power, $(10 \mathrm{~mW} \sim$ $10 \mathrm{~W}$ ) of the power known as the power. The most commonly used medium power calibration system is the combination of "low power meter - directional coupler", in which the directional coupler plays a dual role in power transmission and power coupling, which is the main device that affects the measurement result. In this paper, the coupling parameters of the coupler and the uncertainty components introduced by the reflection parameters are quantitatively analyzed by an example of the power calibration system in a directional coupler. The calculation method of the coupling parameters and the calculation formula of the equivalent reflection coefficient are given.

\section{Introduction to calibration systems}

In this paper, the medium power calibration system discussed in Figure 1, the school power is connected to the directional coupler through the arm, the standard power is connected to the coupling arm, making the system output power P2 (10W) traceable to low power standards (1mW).

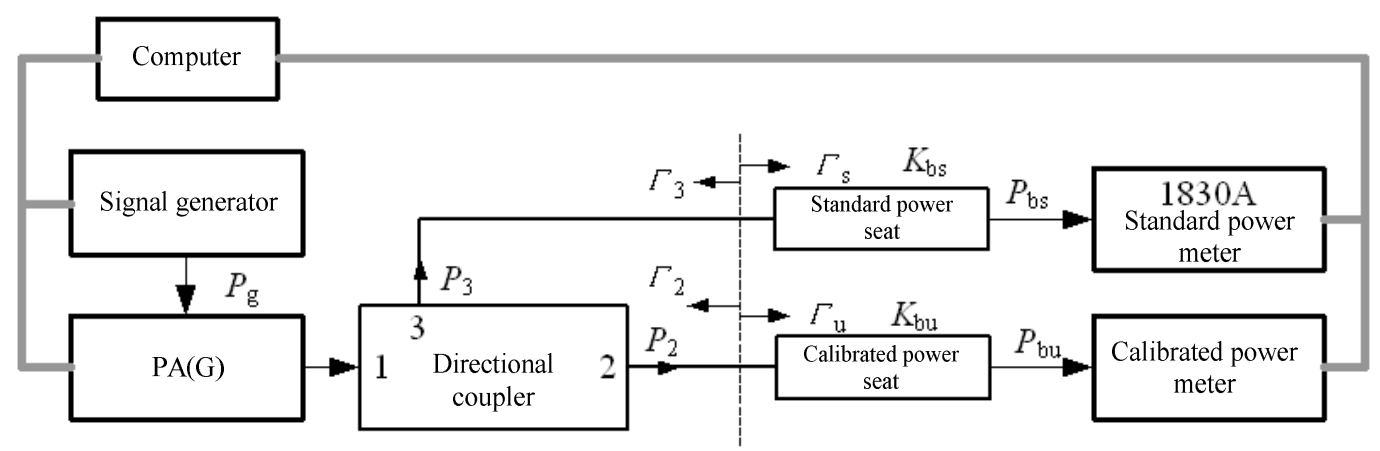

Figure 1. A Medium Power Calibration System Based on Directional Coupler

The calibration result is the correction factor of the school power station. The formula is:

$$
K_{\text {bu }}=K_{\text {bs }} \cdot \frac{P_{\text {bu }}}{P_{\text {bs }}} \cdot K_{32} \cdot \frac{\left|1-\Gamma_{\mathrm{u}} \Gamma_{2}\right|^{2}}{\left|1-\Gamma_{\mathrm{s}} \Gamma_{3}\right|^{2}}
$$

Where $\mathrm{K}_{\mathrm{bu}}$ and $\mathrm{K}_{\mathrm{bs}}$ are the correction factors of the school power station and the standard power block, respectively; $\mathrm{P}_{\mathrm{bu}}$ and $\mathrm{P}_{\mathrm{bs}}$ are the measured values of the school power station and the standard power block, respectively; $K_{32}=P_{3} / P_{2}$ is defined as the directional coupler port 3 output power $\mathrm{P}_{3}$ and port 2 is the ratio of the output power $\mathrm{P}_{2} ; \Gamma \mathrm{u}$ and $\Gamma$ s are the voltage reflection coefficients of the school power station and the standard power block, respectively; $\Gamma_{2}$ and $\Gamma_{3}$ are the equivalent voltage reflection coefficients of the directional coupler port 2 and port 3 , respectively. 


\section{Influence of Coupling Parameters on Calibration Results}

The coupling parameter $\mathrm{K}_{32}$ of the directional coupler in equation (1) is pre-calibrated. Because $K_{32}=P_{3} / P_{2}$, and $\mathrm{P}_{3}$ and $\mathrm{P}_{2}$ are the output power, so $\mathrm{K}_{32}$ shown in Figure 2 directional coupler three-port network model does not have the corresponding $\mathrm{S}$ parameters, cannot be measured directly through the network analyzer, can only be measured indirectly by formula (2):

$$
K_{32}=S_{31} / S_{21}
$$

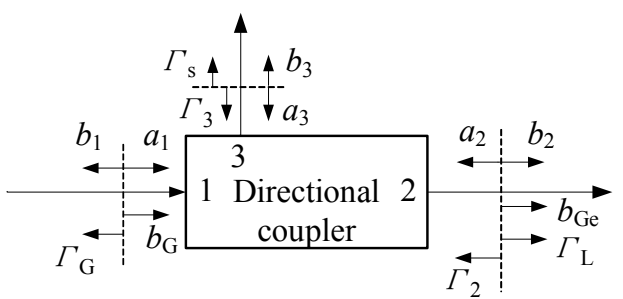

Figure 2. Three - port Network Model of Directional Coupler

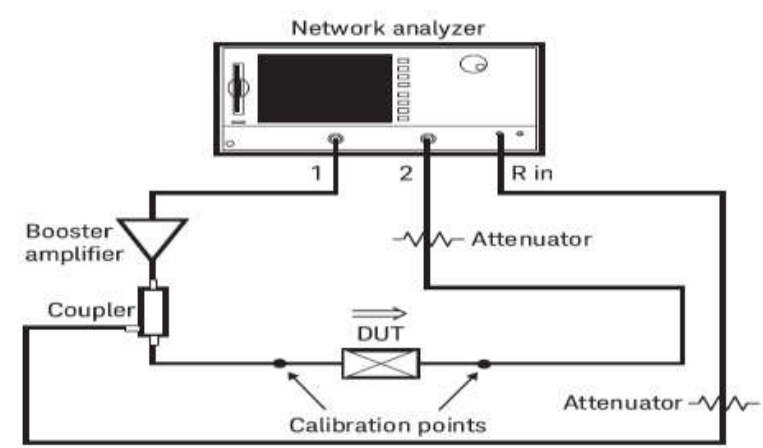

Figure 3. A Power Calibration Method for Directional Couplers

Ideally, $\mathrm{K}_{32}$ should be calibrated at medium power, but requires a special medium power network analyzer, which is difficult for users to obtain; or calibrated with a general network analyzer power extension. [1] provides a calibration method such as Figure 3 shows. The limitation of this method is that only the network analyzer can be calibrated, only the source matching error is corrected. For $10 \mathrm{~W}$ power, the temperature of the directional coupler is very small, so the general laboratory can calibrate the $\mathrm{K}_{32}$ at low power. In this paper, $\mathrm{S}_{31}$ low-power measurement results and Figure 3 shows the power measurement results were compared, as shown in Figure 4.

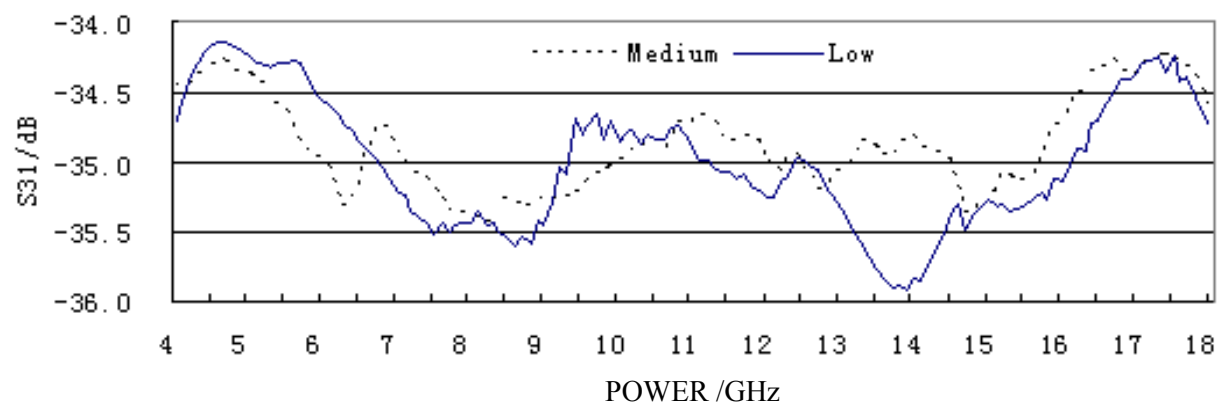

Figure 4. Comparison of Low Power and Medium Power Calibration Directional Couplers

Solid line in the figure is a small power measurement results, the dotted line is the power measurement results, the maximum difference between the two curves $1.1 \mathrm{~dB}$. Taking into account the low power measurement of the network analyzer for the whole two-port calibration, the power measurement is only in response to the calibration, so small power measurement results with a small uncertainty, so this paper select the small power measurement results as directional coupler Calibration data.

According to equation (2), taking into account $S_{31}$ and $S_{21}$ are measured with the same network 
analyzer, so their uncertainty has a strong positive correlation, so the uncertainty is:

If the network analyzer gives the $S_{\mathrm{x} 1}$ maximum measurement error is $\pm 0.1 \mathrm{~dB}$, then:

$u\left(K_{32}\right)=0.1 \mathrm{~dB} / 3 \times 2=0.07 \mathrm{~dB}$

\section{Influence of Reflectance Parameters on Calibration Results}

In equation (1), $\Gamma_{2}$ and $\Gamma_{3}$ are the equivalent voltage reflection coefficients of directional coupler port 2 and port 3, respectively. The scattering and end-of-bound equations for the three-port network shown in Figure 2 are:

$$
\left\{\begin{array}{l}
b_{1}=S_{11} a_{1}+S_{12} a_{2}+S_{13} a_{3} \\
b_{2}=S_{21} a_{1}+S_{22} a_{2}+S_{23} a_{3} \\
b_{3}=S_{31} a_{1}+S_{32} a_{2}+S_{33} a_{3} \\
a_{1}=b_{\mathrm{G}}+b_{1} \Gamma_{\mathrm{G}} \\
a_{2}=b_{2} \Gamma_{\mathrm{L}} \\
a_{3}=b_{3} \Gamma_{\mathrm{s}}
\end{array}\right.
$$

Solve the equation group (3), get:

$$
\Gamma_{2}=S_{22}-\frac{S_{21} S_{32}}{S_{31}}
$$

$\Gamma_{2}$ is the equivalent reflection coefficient of the directional coupler port 2. Similarly, the equivalent reflection coefficient of port 3 can also be obtained. After a series of derivation, you can get:

$$
u(M)=0.85 \sqrt{\left|\Gamma_{\mathrm{u}}\right|^{2}\left|\Gamma_{2}\right|^{2}+\left|\Gamma_{\mathrm{s}}\right|^{2}\left|\Gamma_{3}\right|^{2}}
$$

It is calculated that the maximum uncertainty of the system mismatch is shown in Fig. 1.

\section{The effect of directionality on calibration results}

When the system was tested for repeatability, it was found that the measurement data in the $4 \mathrm{GHz}-18 \mathrm{GHz}$ band changed greatly after each reconnection of the standard power station and the school power station. The maximum change between the groups was $9.5 \%$ and the average change was $5 \% 5$, the data change is much smaller and the maximum change between groups is $0.1 \%$, as shown by the dotted line in Figure 5, when the power sensor is not reconnected.

$418 \mathrm{GHz}$ Connection repeatability test

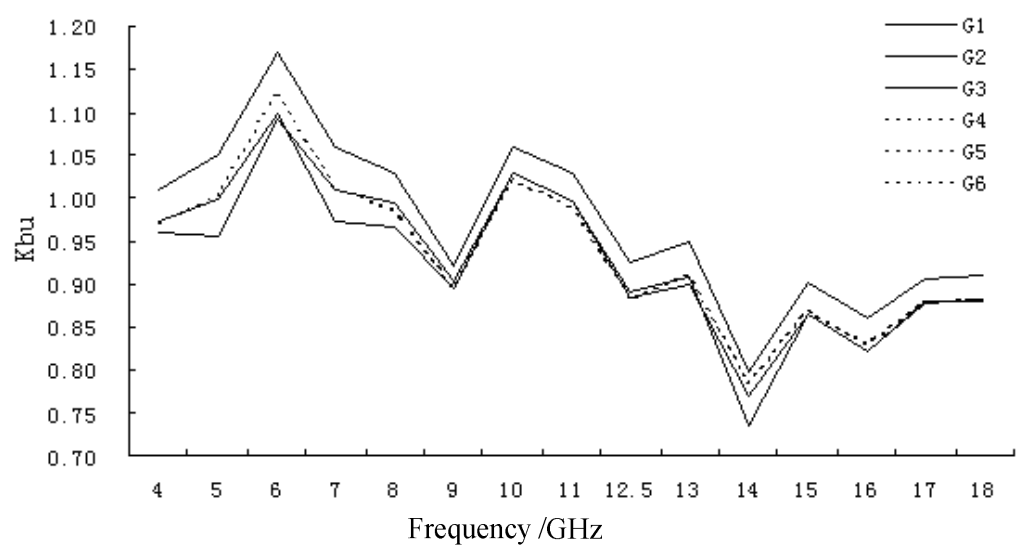

Figure 5. Connection repeatability test

Further analysis revealed that the poor directionality of the $4 \mathrm{GHz}-18 \mathrm{GHz}$ directional coupler increased the dispersion of the measurement data. The net power absorbed by the school power station in Figure 1 can be expressed as:

$$
P_{\mathrm{L}}=P_{2} \frac{1-\left|\Gamma_{\mathrm{u}}\right|^{2}}{\left|1-\Gamma_{2} \Gamma_{\mathrm{u}}\right|^{2}}
$$


It can be seen from the formula (5) that when $\left|\Gamma_{2}\right|$ is large, the smaller phase change between $\Gamma_{2}$ and $\Gamma_{\mathrm{u}}$ will cause a large change in $\mathrm{P}_{\mathrm{L}}$ when reconnecting. Refer to the device manual, Figure 1 in the $4 \mathrm{GHz}-18 \mathrm{GHz}$ band used directional coupler directional value of $16 \mathrm{~dB}$.

\section{Summary}

In this paper, the influence of coupling parameters, reflection parameters and directivity on the measurement results in the medium power calibration system based on directional coupler is analyzed. The coupling parameters and the reflection parameters are typically introduced at $0.07 \mathrm{~dB}$ and $0.1 \mathrm{~dB}$. The poor directivity of the coupler will increase the voltage reflection coefficient of the output port, and its effect is difficult to calculate accurately. The test data show that the maximum change of the measurement result is $9.5 \%(0.8 \mathrm{~dB})$. The test data show that the influence of the directional coupler is negligible when the directivity of the directional coupler is greater than $20 \mathrm{~dB}$.

\section{Reference}

[1]. Key sight Technologies.Using a Network Analyzer to Characterize High-Power Components [R] .5966-3319E.

[2]. State Administration of Quality Supervision, Inspection and Quarantine. JJF1059.1-2012, measurement uncertainty assessment and representation [S] .2012.

[3]. Key sight Technologies, Revisiting Mismatch Uncertainty with the Rayleigh Distribution [R]. 5990-9185EN, 2014.

[4]. Feng Xin Shan, Wu Youzhang, Zhao Ji, etc. High frequency, microwave power measurement test [M]. Beijing: China Metrology Publishing House, 1988. 